\title{
Demo: Dyslexic and Private Reader
}

\section{An Eye-tracking Platform for Reading Interactions with Applications to Increase Empathy and Privacy}

\author{
Kirill Ragozin \\ Keio University, Japan \\ ragozinkirill@gmail.com
}

\author{
Kai Kunze \\ Keio University, Japan \\ kai@kmd.keio.ac.jp
}

\begin{abstract}
Societies all over the world name places in similar ways. Quite often there is no official naming ceremony but places sdmc sn ad called names as points of reference by people. Then an organized body rsdor hm 'mc fhudr the place a name. Frequently it happens that a place has two names: Nmd hr m`ldc ax the people and the other by the government. As in many areas, old g'ahsr chdc hard, and the place continues to be called by its unofficial name long after the meaning is lost.
\end{abstract}

Figure 1: Dylexic Reader Concept: Making it harder to read text by simulating the experience of a person having a specific type of dyslexia.

\begin{abstract}
We present a demonstration based on a MobileHCI 2019 paper to use eye gaze to selectively render or obscure text. Obscuring is used to "simulate" a specific kind of dyslexia. Selectively render text is to give users a more private reading experience in public spaces.
\end{abstract}

\section{KEYWORDS}

datasets, neural networks, gaze detection, text tagging

\section{ACM Reference Format:}

Kirill Ragozin and Kai Kunze. 2019. Demo: Dyslexic and Private Reader: An Eye-tracking Platform for Reading Interactions with Applications to Increase Empathy and Privacy. In Adjunct Proceedings of the 2019 ACM International foint Conference on Pervasive and Ubiquitous Computing and the 2019 International Symposium on Wearable Computers (UbiComp/ISWC '19 Adjunct), September 9-13,

Permission to make digital or hard copies of all or part of this work for personal or classroom use is granted without fee provided that copies are not made or distributed for profit or commercial advantage and that copies bear this notice and the full citation on the first page. Copyrights for components of this work owned by others than ACM must be honored. Abstracting with credit is permitted. To copy otherwise, or republish, to post on servers or to redistribute to lists, requires prior specific permission and/or a fee. Request permissions from permissions@acm.org.

UbiComp/ISWC '19 Adjunct, September 9-13, 2019, London, United Kingdom (c) 2019 Association for Computing Machinery.

ACM ISBN 978-1-4503-6869-8/19/09 ..\$15.00

https://doi.org/10.1145/3341162.3343788
2019, London, United Kingdom. ACM, New York, NY, USA, 2 pages. https://doi.org/10.1145/3341162.3343788

\section{INTRODUCTION}

Reading together with writing is one our most significant technological inventions. However, digital reading hasn't yet changed much compared to the offline version. We are working on research to incorporate the user's current state into the reading experience [1-3].

This demonstration of a platform used for an accepted full paper at MobileHCI titled "Private Reader: Using Eye Tracking to Improve Reading Privacy in Public Spaces." We developed Private Reader, a method to reduce comprehension, increase frustration for the observer yet maintaining readability for the user (not impacting the reading speed). Compared to the publication, we present not only the Private Reader modes to improve privacy but also Dyslexic Reader, a mode making it harder to read to experience a specific kind of dyslexia, in which a person has a hard time focusing on words as it seems to them the letters jump and change position [4].

\section{DEMONSTRATION}

We use eye tracking to render only the word a user is currently reading as a core concept. Current commodity eyetracking devices cannot provide this level of accuracy; we need to also change a couple of words around the user's gaze. 


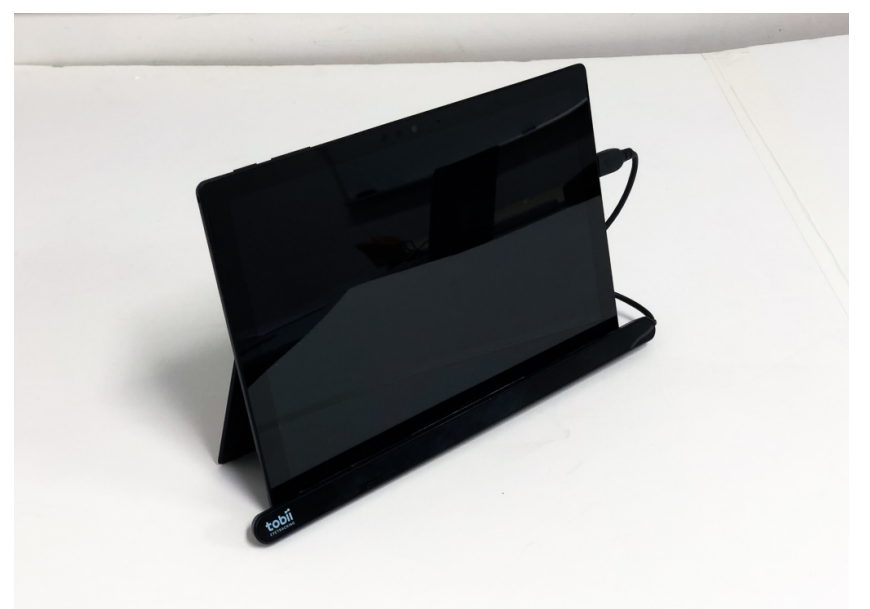

Figure 2: The demonstration system. A Windows tablet with Tobii 4C attached.

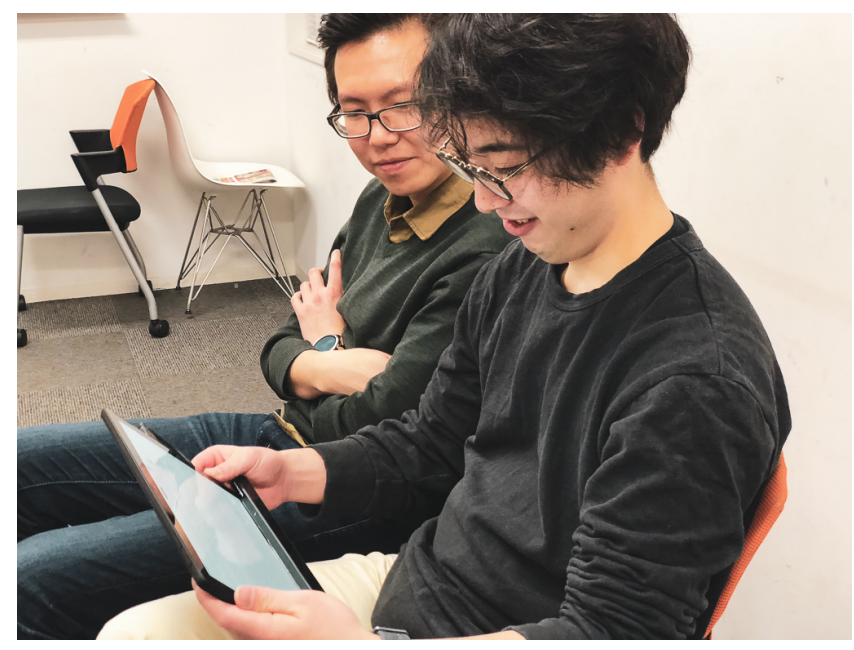

Figure 3: A user reading on the system, while an observer trying to sholder surf.

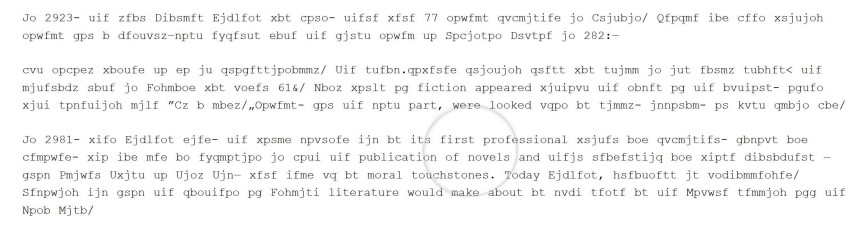

Figure 4: The private reader mode. The bubble shows the current eyegaze position of the user and is just added for illustration purposes
We present two basic applications: Dyslexic and Private Reader. Dyslexic Reader renders part of the words a user is trying to read into gibberish, making it harder for the user to read the text. This is to simulate a specific type of dyslexia and is to help users to better understand what it means to have this disability.

Private Reader renders text outside of a gaze bubble as scrambled text, where a word looks like a collection of random letters.

Figure 2 shows the prototype system implemented and used for the user studies. We use a Windows Surface Pro 6 Tablet with a Tobii $4 \mathrm{C}$ eye tracker attached. We obtained a Tobii $4 \mathrm{C}$ research license to use the system for this publication. The Tobii $4 \mathrm{C}$ is a standard commercial eye tracker with an approximate sampling rate of $60 \mathrm{~Hz}$ for the gaze data.

Figure 3 shows a user reading with the system and an observer looking over the shoulder.

Figure 4 gives you a glimps on the Private Reader mode (compared to Dyslexic Reader in Figure 1). Only the words in the gaze bubble are displayed correctly the other words are scrambled and flash with the users reading speed.

The users will have the possibility to try out both Dyslexic and Private Reader, an interaction (explaining the concept and reading a couple of lines) usually takes about 2-3 minutes per person.

We are looking for collaborators to share our technology and continuing with the development. The code for Dyslexic and Private Reader will be open-sourced.

\section{ACKNOWLEDGMENTS}

This work was partly supported by the JST CREST Project on Experiential Supplements, Grant No. JPMJCR16E1.

\section{REFERENCES}

[1] Olivier Augereau, Charles Lima Sanches, Koichi Kise, and Kai Kunze. 2018. Wordometer systems for everyday life. Proceedings of the ACM on Interactive, Mobile, Wearable and Ubiquitous Technologies 1, 4 (2018), 123.

[2] Kai Kunze, Masakazu Iwamura, Koichi Kise, Seiichi Uchida, and Shinichiro Omachi. 2013. Activity recognition for the mind: Toward a cognitive" Quantified Self". Computer 46, 10 (2013), 105-108.

[3] Kai Kunze, Yuzuko Utsumi, Yuki Shiga, Koichi Kise, and Andreas Bulling. 2013. I know what you are reading: recognition of document types using mobile eye tracking. In Proceedings of the 2013 International Symposium on Wearable Computers. ACM, 113-116.

[4] G Reid Lyon, Sally E Shaywitz, and Bennett A Shaywitz. 2003. A definition of dyslexia. Annals of dyslexia 53, 1 (2003), 1-14. 